\title{
An electrically switchable viewing angle device based on dichroic dyes doped polymer stabilized liquid crystals Le Zhou ${ }^{1,2}$, Shuaifeng Zhang ${ }^{3}$, Tingjun Zhong ${ }^{4}$, Lanying Zhang ${ }^{2^{*}}$
}

1 School of Materials Science and Engineering, Tsinghua University, Beijing, People's republic of China; zhoule@pku.edu.cn

2 Key Laboratory of Polymer Chemistry and Physics of Ministry of Education; School of Materials Science and Engineering, College of Engineering, Peking University, Beijing, People's Republic of China;

3 Department of Materials Physics and Chemistry, School of Materials Science and Engineering, University of Science and Technology Beijing, Beijing, People's Republic of China; shuaifengustb@163.com

4 Department of Chemistry, College of Science, China Agricultural University, Beijing, People's republic of China; zhongtingjunjun@126.com

* Corresponding author: zhanglanying@pku.edu.cn 
Abstract: Accompanying with the fast development of modern technologies, computers and cellphones are applied in people's work and life, which brings more convenience and fun. However, due to the wide viewing angle (WVA) characteristics of these electronic products, people can be peered by others when they glaze over the materials, which leads to the leakage of personal privacy and commercial secrets. Consequently, liquid crystal displays (LCDs) simultaneously with narrow viewing angle (NVA) characteristics are needed to avoid the risk of secret leakage, thus electrically switchable viewing angle devices are attracting considerable attention because of the information protection. Herein, we have developed an electrically switchable viewing angle device that fabricated by dichroic dyes doped polymer stabilized cholesteric liquid crystals (DD-PSCLCs). By fixing the pitch of CLCs at the infrared light region, when CLCs are at the planar state, the device is transparent at zero field. When applying the electric field, CLCs are switched to the focal conic texture, thus the device is lightscattered. Therefore, the fabricated device can make the viewing angle of LCDs electrically switchable between WVA mode and NVA mode by small voltage, which makes contribution to privacy protection.

Keywords: Liquid crystals; Dichroic dyes; Viewing angle; Polymer stabilized liquid crystals; Privacy protection 


\section{Results and Discussion}
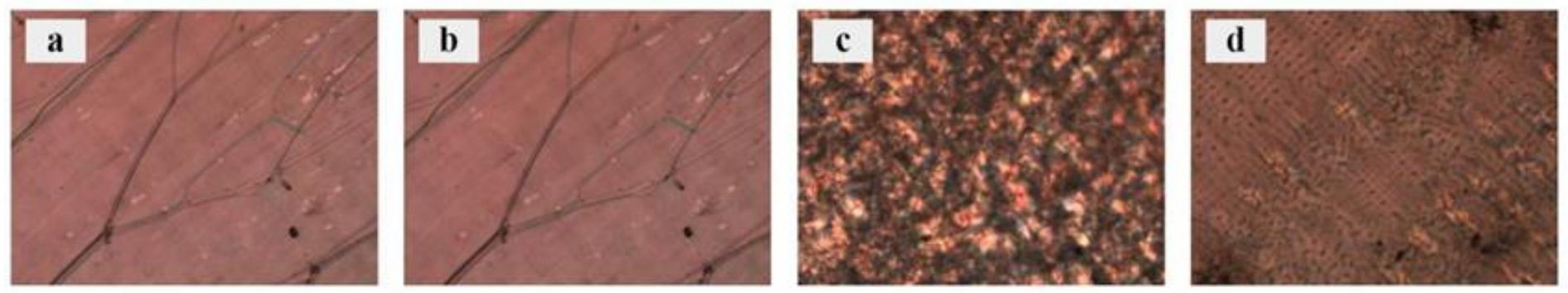

Figure 1. Electrically switchable polarized optical microscope (POM) images of DD-PSCLC film after UV polymerization. Figure 1 (a) illustrates the texture of DD-PSCLC film at the planar state without electric field. When a $10 \mathrm{~V}$ voltage is applied to the film, CLCs are still in planar state, the texture of the film is presented in Figure 1 (b). Continually increasing the applied voltage of electric field, the film turns to be focal conic, which is shown in Figure 1 (c). When the applied voltage of electric field is $30 \mathrm{~V}$, CLCs in the DD-PSCLC film are unwound, the non-UV-irradiated part turns to be transparent, as depicted in Figure 1 (d).

\section{Conclusions}

We have successfully demonstrated a controllable viewing angle device based on DDPSCLC film, whose viewing angle can be switched between two states, WVA mode and NVA mode. Without electric field, CLCs in the DD-PSCLC film are planar aligned, the device is in WVA mode. By applying small voltage, CLCs in the DD-PSCLC film are in the focal conic state, the device is in WVA mode, the optical property in the WVA mode is $\pm 60^{\circ}$ of the visible angles. If the applied voltage is over than $\mathrm{E}_{\mathrm{C}}$ CLCs in the DD-PSCLC film turn homeotropic, the device presents NVA mode, the optical property for the NVA mode is $\pm 30^{\circ}$ of the visible angles. 


\section{Supplementary Materials}
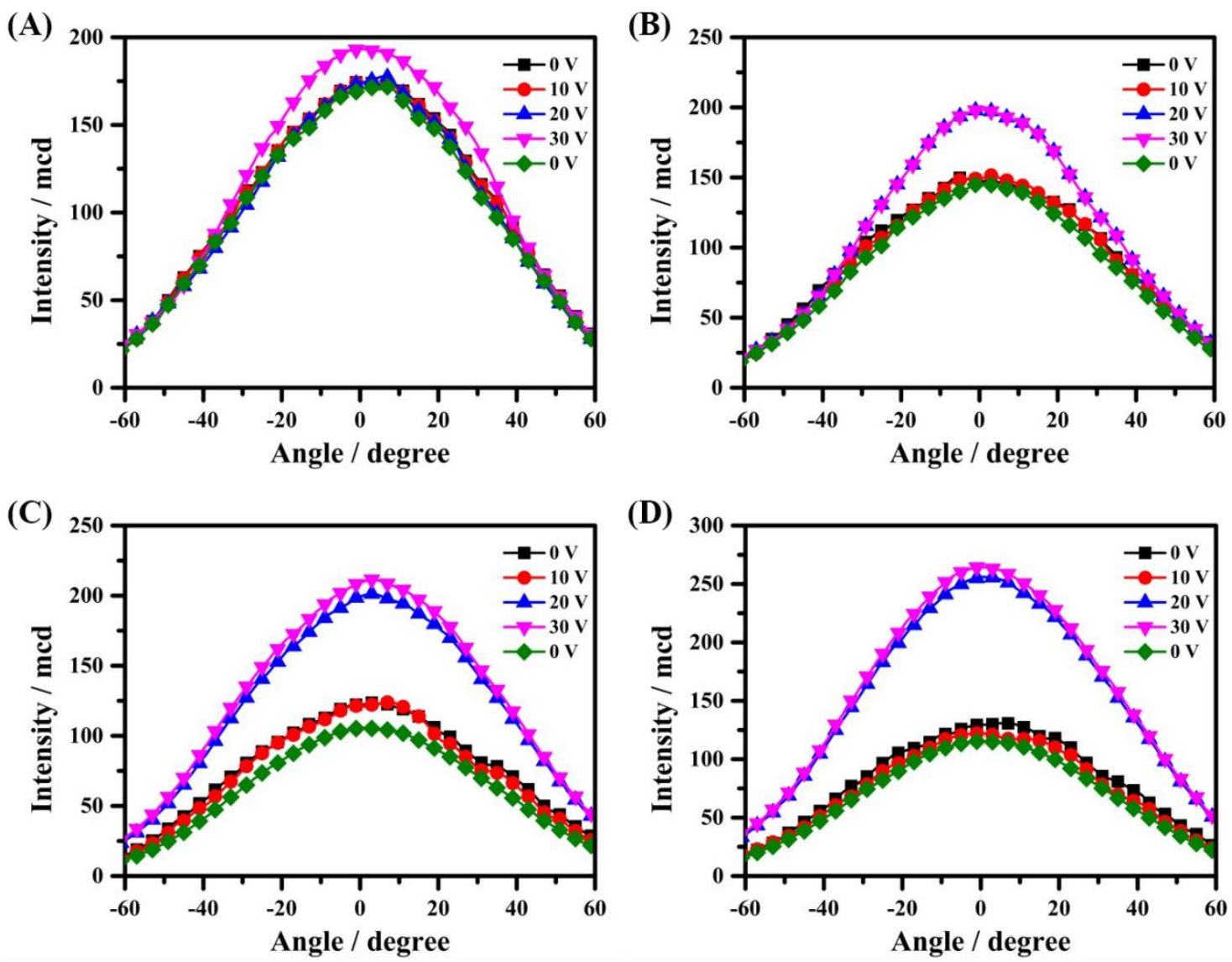

Figure 2. The angle-intensity characteristics of viewing angle controllable device by fabricating patterned polymer with varied dimensions. (A) 40-10 $\mu \mathrm{m}$; (B) 40-20 $\mu \mathrm{m}$; (C) $40-40 \mu \mathrm{m}$; (D) 40-60 $\mu \mathrm{m}$. 


\section{Supplementary Materials}

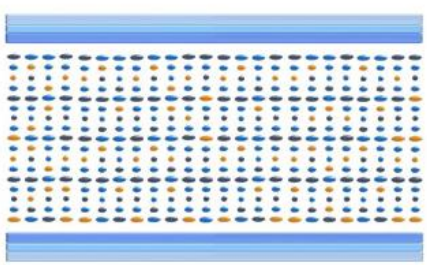

(A)

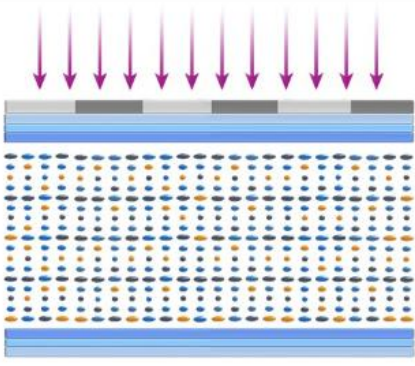

(B)

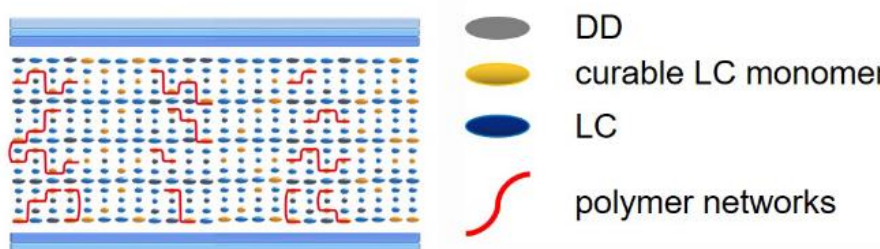

(C)

Figure 3. The process for preparing DD-PSCLC film. (A) DDs and CLCs are in the planar state, as the upper PET substrate and the lower PET substrate are both coated with PVA planar alignment layer; (B) The film is UV irradiated under photo-mask with different dimensions; (C) The patterned polymer network has formed in the film.

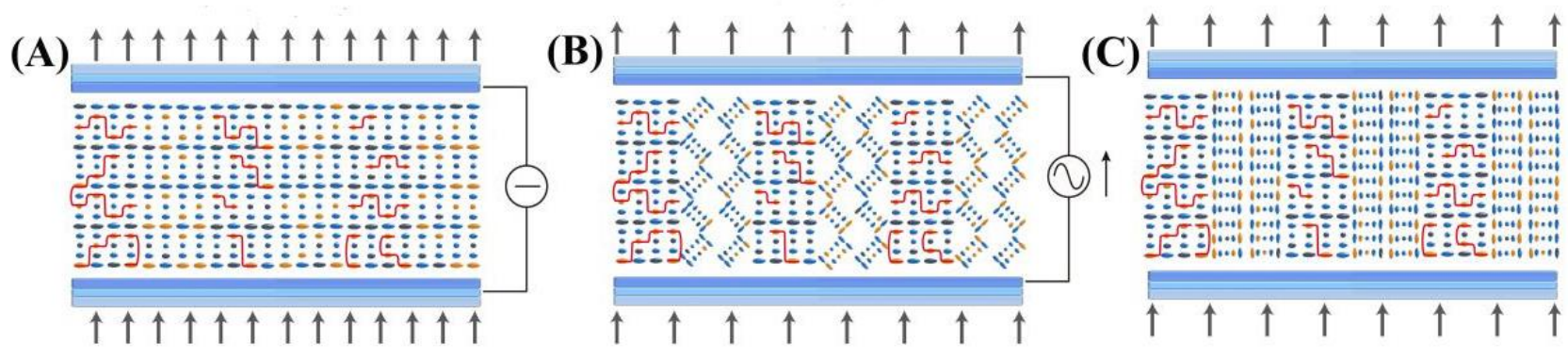

Figure 4. The switchable mechanism of DD-PSCLC film. (A) The planar state; (B) The focal conic state (WVA mode); (C) The homeotropic state (NVA mode). 


\section{Acknowledgments}

Author Contributions: Conceptualization, Le Zhou; methodology, Le Zhou; software, Shuaifeng Zhang and Tingjun Zhong; writing-review and editing, Lanying Zhang; supervision. All authors have read and agreed to the published version of the manuscript.

Funding: This research was funded by the National Key R\&D Program of China (2018YFB0703704, 2019YFC1904702), the National Natural Science Foundation of China (NSFC) (Grant no. 51921002 and 51927806), the joint fund of the ministry of education for equipment pre research (6141A020222). 